\title{
Spin-polarized transport in ferromagnetic multilayered semiconductor nanostructures
}

\author{
E. J. R. Oliveira \\ Instituto de Física, Universidade do Estado do Rio de Janeiro, 20.500-013 Rio de Janeiro, R.J., Brazil \\ A. T. da Cunha Lima \\ Universidade Veiga de Almeida, Campus de Cabo Frio, 28905-970 Cabo Frio, RJ, Brazil \\ M. A. Boselli \\ Departamento de Física, Universidade do Federal de Ouro Preto, 35400-000 Ouro Preto, M.G., Brazil \\ G. M. Sipahi \\ Instituto de Física de São Carlos, Universidade de São Paulo, CP 369, 13560-970, São Carlos, SP, Brazil \\ S. C. P. Rodrigues \\ Departamento de Física, Universidade Federal de Pernambuco, 50670-901 Recife, PE, Brazil \\ I. C. da Cunha Lima \\ Instituto de Física, Universidade do Estado do Rio de Janeiro, 20.500-013 Rio de Janeiro, R.J., Brazil, and \\ Departamento de Física, Universidade do Federal de Ouro Preto, 35400-000 Ouro Preto, M.G., Brazil
}

(Dated: May 1, 2017)

\begin{abstract}
The occurrence of inhomogeneous spin-density distribution in multilayered ferromagnetic diluted magnetic semiconductor nanostructures leads to strong dependence of the spin-polarized transport properties on these systems. The spin-dependent mobility, conductivity and resistivity in (Ga,Mn)As/GaAs, $(\mathrm{Ga}, \mathrm{Mn}) \mathrm{N} / \mathrm{GaN}$, and $(\mathrm{Si}, \mathrm{Mn}) / \mathrm{Si}$ multilayers are calculated as a function of temperature, scaled by the average magnetization of the diluted magnetic semiconductor layers. An increase of the resistivity near the transition temperature is obtained. We observed that the spinpolarized transport properties changes strongly among the three materials.
\end{abstract}

Spin-polarized current and inhomogeneous spin density are two elements to consider in Spintronics. During the last years the fabrication and characterization of diluted magnetic semiconductor (DMS) nanostructures has rapidly evolved. In the particular case of DMS based on GaAs, GaN, ZnO, Si, and a few other semiconductors a ferromagnetic phase exists with transition temperatures near and even above room temperature [1, 2]. Carriers in high concentration present in these systems play an important role in the existence of the magnetic order. Ferromagnetism has been shown to persist in some multilayered diluted magnetic heterostructures (MDMH), structures with alternating non-magnetic and magnetic layers [3, 4] which produce an inhomogeneous spin polarization density. In these MDMH the carriers come out of the ionized magnetic atoms acting as donors or acceptors, even when some compensation mechanism occur. In such structures the states are spin-polarized and carriers with distinct spin-polarization are scattered by the ionized atoms differently. Consequently, in the ferromagnetic phase MDMHs show both spin-polarized currents and inhomogeneous spin densities.

We present a calculation of the spin-polarized transport properties of $(\mathrm{Ga}, \mathrm{Mn}) \mathrm{As} / \mathrm{GaAs},(\mathrm{Ga}, \mathrm{Mn}) \mathrm{N} / \mathrm{GaN}$, and $(\mathrm{Si}, \mathrm{Mn}) / \mathrm{Si} \mathrm{MDMH}$ as a function of temperature. The aim of this paper is to explore the interplay the presence of the magnetization and the spin-dependent scattering processes as temperature changes. We observe how conductivity, resistivity, and mobility for each spinpolarization are affected as the sample average magnetization changes with temperature. It has been shown [5] that an inhomogeneity in the spin density of ferromagnetic MDMH structures is responsible for the high transition temperatures observed.

We start with a model assuming a heterostructure in which: (i) scattering centers occur only in certain layers, and they are the very source of carriers; (ii) these layers are separated by undoped spacers, ideally without any band mismatch; (iii) the carriers are assumed to be in the metallic regime; (iv) barriers represented by the potential $U_{\text {mod }}$ in the growth direction are added to the layers, either to the spacers or to the doped layers, having their height modulated by some external parameter. Later we will relate them to the sample temperature. Carriers are attracted to the doped layers by the Coulomb interaction with the ionized impurities and are also affected by the confining potential represented by the parametric barriers. In the case of high carrier concentration a simplified model can be used to obtain the electronic structure assuming that the charged impurities are substituted by a homogeneous background, in a kind of jellium model.

The Hamiltonian of the motion in the $z$-direction reduces to that of a free particle of effective mass $m^{*}$ plus terms due to the confinement, $U_{\bmod }(z)$ and $V_{C}(z)$. 
(a)
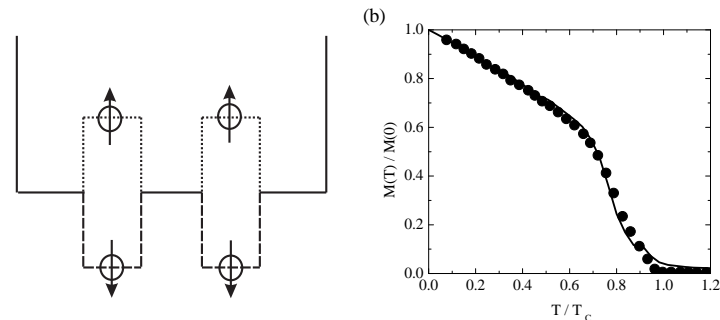

FIG. 1: (a)Effective magnetic barriers for holes with spins aligned (up) and anti-aligned (down) to the average magnetization. (b)Modulation function for the potential barrier extracted from the curve of the magnetization versus temperature. Solid line after Ref 3 , dots after Ref 4 .

The latter incorporates the Hartree and the exchangecorrelation terms of the carrier-carrier interaction, and the interaction carrier-background:

$$
\left[-\frac{\hbar^{2}}{2 m^{*}} \frac{\partial^{2}}{\partial z^{2}}+U_{\bmod }(z)+V_{C}(z)\right] \Psi_{n}(z)=E_{n} \Psi_{n}(z) .
$$

The subbands are parabolic and have their energy given by $E_{n}(k)=\hbar^{2} k^{2} / 2 m^{*}+E_{n}$, with $\mathbf{k}=\left(k_{x}, k_{y}\right)$. Eq. (10) and the Poisson equation are solved self-consistently.

Next we calculate the in-plane transport relaxation time due to scattering by ionized impurities based on the linearized Boltzmann equation, as described in Ref.(岩). We neglect inter-subband transitions. The current is obtained by summing up the contribution of all occupied subbands, which behave as different conducting channels. The mobility of each channel is modulated by the barrier height, since it determines not only the Fermi wavevectors of each subband, $k_{F}^{(n)}$, but also the probability densities $\left|\Psi_{n}(z)\right|^{2}$, affecting directly the form factor for an impurity located at $z=z_{i}$ :

$$
\left.g_{\mathrm{imp}}^{(n)}\left(\theta, z_{i}\right)=\int_{-\infty}^{\infty}\left|\Psi_{n}(z)\right|^{2} \exp \left[-k_{n}(\theta)\left|z-z_{i}\right|\right]\right),
$$

where $\theta$ is the scattering angle and we used $k_{n}(\theta)=$ $2 k_{F}^{n} \sin \frac{\theta}{2}$. The inverse of the momentum relaxation time for subband $n$, using the screening form factor $g_{s}(q)$ as in Ref. 6, results in:

$$
\begin{aligned}
& \frac{1}{\tau}_{n}=\frac{2 e^{2} m^{*} n_{i}}{\hbar^{3} \kappa} \int_{0}^{\pi} d \theta(1-\cos \theta) \times \\
& {\left[k_{n}(\theta)+q_{0} g_{s}\left(k_{n}(\theta)\right)\right]^{-2} \int d z g(z) g_{\mathrm{imp}}^{(n)}(\theta, z),}
\end{aligned}
$$

where $\kappa$ is the dielectric constant, $n_{i}$ the ionized impurity concentration in each DMS layer, $q_{0}=n_{s} / a_{0}$, with $n_{s}$ representing the effective areal carrier concentration, $a_{0}$ the effective Bohr radius, and $g(z)$ equals 1 if $z$ lies in the magnetic layer and zero otherwise.

The in-plane transport of MDMHs can be mapped into this model. In mean field approximation an average magnetization $\langle M\rangle$ of the magnetic layers provides a potential barrier which is $\pm 75 \mathrm{meV}$ for $\mathrm{Ga}_{0.95} \mathrm{Mn}_{0.05} \mathrm{As}$ at
$\mathrm{T}=0 \mathrm{~K}$, as shown in Fig. 1h. Free carriers are heavy holes. It has been observed that in MDMH structures the magnetization changes with temperature according to the curve shown in Fig. 10. This curve is used here as a scale relating the temperature to the magnetic barriers. This Ansatz provides the dependence of the spindependent modulated potential $U_{\bmod }(z)$ in Eq.(1) with the sample temperature. It contains the complete information about the magnetic part of the system. On the other hand, the calculation of the transport relaxation time depends on the concentration of ionized impurities, which is taken as equal to the carrier concentration. The limit $T=0$ is assumed for the equilibrium distribution in the linearized Boltzmann equation, but a finite $T$ is taken as a parameter modulating the barrier height. In this sense, we define a normalized conductivity $\sigma(T) / \sigma(0)$ as the ratio between the conductivities resulting of the average magnetization at temperature $T$, and the maximum magnetization obtained when $T=0$. We can also obtain the fraction of the normalized conductivity coming out of spins aligned (up) or anti-aligned (down) to the average magnetization by summing up the contributions of the channels with equal spin polarization.

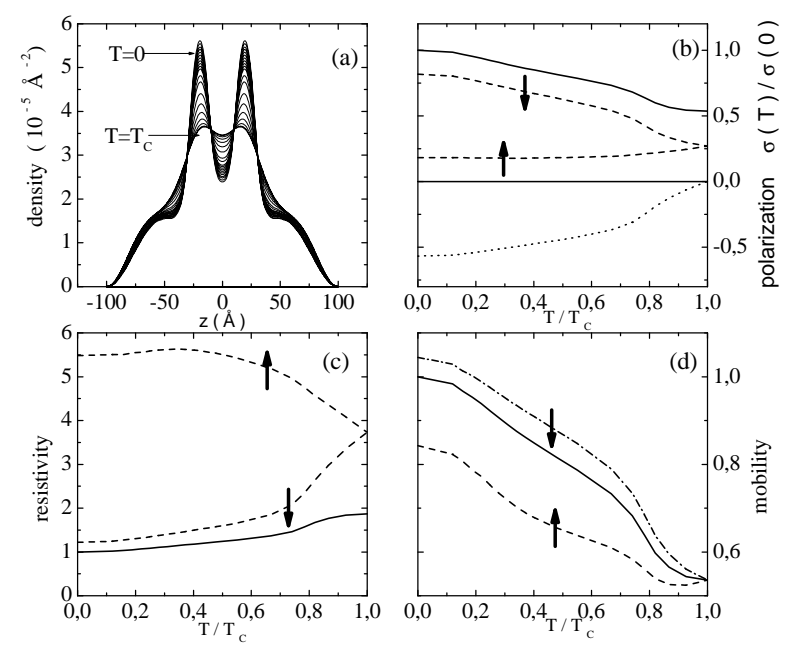

FIG. 2: (Ga,Mn)As/GaAs MDMH: (a) charge density distribution; (b) normalized conductivities and spin- polarization; (c) spin-polarized and total resistivities; (d) spin-dependent and total mobility. Total quantities are indicated by full lines, spin-dependent quantities by dashed lines with arrows indicating parallel $(\uparrow)$ or anti-parallel $(\downarrow)$ polarization.

The calculations were performed for (Ga,Mn)As/GaAs, (Ga,Mn)N/GaN, and $(\mathrm{Si}, \mathrm{Mn}) / \mathrm{Si}$. In each case we calculated (a) the areal charge densities as a function of the position in the z-axis for different temperatures; (b) the normalized spin-dependent and total conductivity together with the polarization; (c) the spin-polarized and total resistivities; (d) the spin-polarized and total mobilities. 

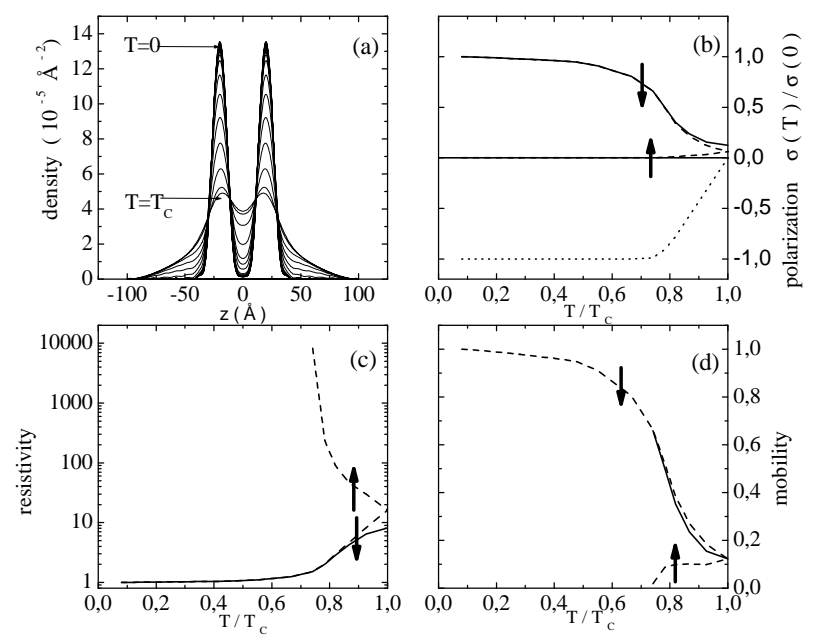

FIG. 3: For (Ga,Mn)N/GaN, as above, using a logarithmic scale for the resistivity.
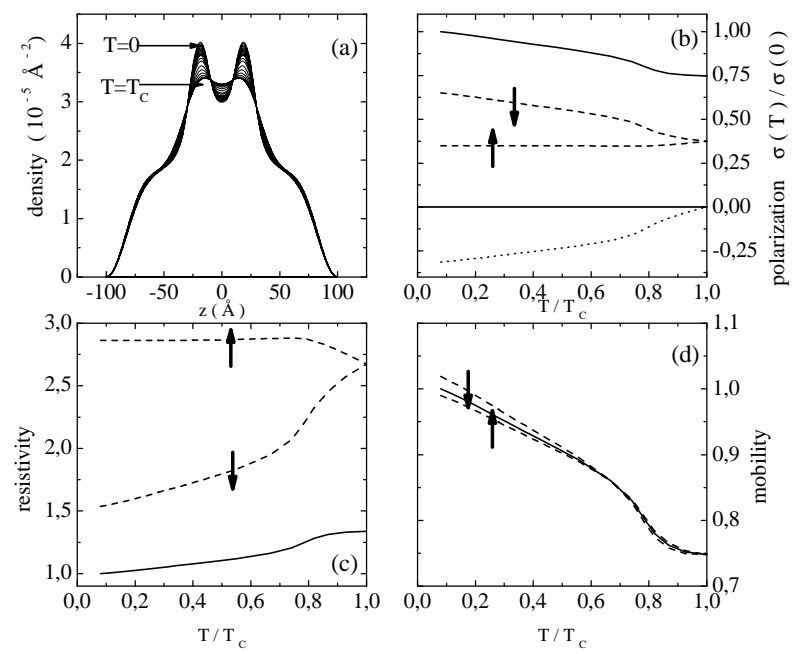

FIG. 4: Same as above for $(\mathrm{Si}, \mathrm{Mn}) / \mathrm{Si}$

The carrier concentration inside the DMS layers decreases as the temperature increases, showing that the magnetic barriers compete with the Coulomb attraction due to ionized atoms at low temperature, but loses importance as the temperature increases, as expected. Observing Figs. 2(a), 3(a) and 4(a) we see that, in the case of $(\mathrm{Ga}, \mathrm{Mn}) \mathrm{N} / \mathrm{GaN}$ the concentration of the charge density inside the DMS layers is almost complete, even at temperatures near $T_{C}$. For $(\mathrm{Si}, \mathrm{Mn}) / \mathrm{Si}$ the concentration is the weakest among the three systems. As a consequence of the strength of the magnetic barriers, carriers in $(\mathrm{Ga}, \mathrm{Mn}) / \mathrm{GaN}$ are completely spin-polarized even close to $T_{C}$. The polarization is weaker for $(\mathrm{Ga}, \mathrm{Mn}) \mathrm{As} / \mathrm{GaAs}$, and weaker still for (Si,Mn)Si.

The conductivity for each spin-dependent channel depends not only on the transport relaxation time, but also on the density of carriers occupying the subband. We show in Figs. 2(b)-4(b) the normalized conductivity, defined as $\sigma(T) / \sigma\left(T_{C}\right)$. For (Ga,Mn)As/GaAs and $(\mathrm{Ga}, \mathrm{Mn}) \mathrm{N} / \mathrm{GaN}$, there is a remarkable difference between the conductivity for channels with spins up and down. For $(\mathrm{Si}, \mathrm{Mn}) / \mathrm{Si}$, with full spin-polarization, the conductivity is zero for spins up until very near $T_{C}$. The resistivities, shown in Figs 2(c)-4(c), reflect the behavior of the conductivity. It is worthwhile to stress the fact that a hump appears near $T_{C}$, corresponding to the increase of the resistivity as the transition temperature is aproached. This behavior was observed in $(\mathrm{Ga}, \mathrm{Mn}) \mathrm{As}$ epilayers [1], and is a consequence of the change in the subbands population as the magnetic barriers decrease drastically when the transition temperature is reached. Finally, it is important to obtain the mobility, since it is directly related to the average velocity of carriers with a given spin-polarization. While the mobility in $(\mathrm{Si}, \mathrm{Mn}) / \mathrm{Si}$ is practically spin-independent, in $(\mathrm{Ga}, \mathrm{Mn}) \mathrm{As} / \mathrm{GaAs}$ the difference is almost $30 \%$ for spins up and down. For $(\mathrm{Ga}, \mathrm{Mn}) \mathrm{N} / \mathrm{GaN}$ we observe a kind of half-metallic behavior, where carriers with spins up have very small mobility until very near to $T_{C}$, while carriers with spin down are metallic.

Besides the fact that our calculation explains the hump in the resistivity near $T_{C}$, we have shown that the charge density and spin density inhomogeneities resulting of the effective magnetic barriers in MDMH can be used to provide highly spin-polarized resistivity and mobility. Certainly, spin-flip mechanisms as well as interaction with phonons must be included if a more complete treatment near the transition temperature is desired. However, we believe that MDMH are useful at a temperature range far enough from $T_{C}$ to make our calculation realistic.

This work was partially supported by CNPq (ESN fellowship and research grant), FAPEMIG, FAPESP and FAPERJ. ICCL is grateful for the hospitality of Prof. M. W. Wu group at the USTC, Hefei, Anhui, China.

[1] F. Matsukura, H. Ohno, A. Shen, and Y. Sugawara, Phys. Rev. B 57, R2037 (1998); D. Chiba, K. Takamura, F. Matsukura, and H. Ohno, Appl. Phys. Lett. 82, 3020 (2003).

[2] T. Dietl, H. Ohno, and F. Matsukura, Phys. Rev. B 63, 195205 (2001).

[3] M. A. Boselli, I. C. da Cunha Lima, J. R. Leite, A. Troper, and A. Ghazali, Appl. Phys. Lett. 84, 1138 (2004).

[4] R. Mathieu, P. Svendlindh, J. Sadowski, K. Swiatek, M. Karlsteen, J. Kanski, and L. Ilver, Appl. Phys. Lett. 81, 3013 (2002).

[5] M. A. Boselli, I. C. da Cunha Lima, and A. Ghazali, Phys. Rev. B 68, 085319 (2003).

[6] Wave Mechanics Applied to Semiconductor Heterostructures, pp. 193-219, by G.Bastard, Halsted Press, John Wiley, New York (1988). 